\title{
Synergistic protective effect of a BN-carbon separator for highly stable lithium sulfur batteries
}

\author{
Patrick Joo Hyun Kim${ }^{1}$, Jihoon $\mathrm{Seo}^{1}$, Kun $\mathrm{Fu}^{2}$, Junghyun $\mathrm{Choi}^{1}$, Zhiming Liu ${ }^{1}$, Jiseok Kwon ${ }^{1}$, Liangbing $\mathrm{Hu}^{2}$ \\ and Ungyu Paik ${ }^{1}$
}

Lithium sulfur (Li-S) batteries have drawn much attention as next-generation batteries because of their high theoretical capacity $\left(1672 \mathrm{mAh}^{-1}\right)$, environmental friendliness and low cost. However, several critical issues, which are mainly associated with the polysulfide shuttling effect, result in their poor electrochemical performance. Carbon-modified separators have been introduced to attempt to address these systemic challenges. However, this approach focused only on the suppression of dissolved polysulfides on the cathodic side without considering the further entrapment of polysulfides on the anodic side. In this study, we first designed a multifunctional trilayer membrane comprising a carbon layer and a boron nitride (BN) layer to facilitate the electrochemical performance of Li-S batteries and protect the $\mathrm{Li}$ anode from unexpected side reactions. When a BN-carbon separator was employed, the sulfur cathode delivered stable capacity retention over $\mathbf{2 5 0}$ cycles and an excellent specific capacity $\left(702 \mathrm{mAh} \mathrm{g}^{-1}\right)$ at a high current density $(4 \mathrm{C})$. The BN-carbon separator also facilitated the uniform plating/striping of $\mathrm{Li}$ and, thus, suppressed the severe growth of dendritic $\mathrm{Li}$ on the electrode; this led to the stable operation of the $\mathrm{Li}$ anode with a high Coulombic efficiency and improved cycling performance.

NPG Asia Materials (2017) 9, e375; doi:10.1038/am.2017.51; published online 14 April 2017

\section{INTRODUCTION}

Advanced electronic devices, such as cell phones and laptops, play crucial roles in our lives, and interest in developing affordable, advanced energy storage systems with high-energy densities has increased dramatically. ${ }^{1-3}$ In response to the recent trend of developing high-energy lithium-ion batteries, significant efforts have been devoted to utilizing sulfur as a cathode material because of its high theoretical capacity $\left(1672 \mathrm{mAhg}^{-1}\right)$, positive cost:benefit ratio, and environmental benignity. ${ }^{2,4-7}$ However, an inherent problem of Li-S batteries is the dissolution of polysulfides in the electrolyte, which causes critical issues, including low Coulombic efficiency, capacity fading, Li corrosion and self-discharging. ${ }^{2,4,8}$ These challenges will hinder the commercialization of sulfur cathodes in Li-S batteries. To address these challenges, many designs based on carbon-sulfur composite structures have been explored, including: (a) impregnation of sulfur species inside the mesoporous scaffold, ${ }^{1,9-11}$ (b) compact encapsulation of the sulfur electrode with functional polymers, ${ }^{12-15}$ and (c) carving of hydrophilic groups (for example, polyethylene glycol and polyacrylic acid) on the sulfur reservoir. ${ }^{16}$ These strategies have provided significant improvements in alleviating the polysulfide shuttling effect; however, preparing carbon-sulfur composites is often expensive and requires significant effort, which potentially hinders the production of sulfur cathodes for Li-S batteries on a commercial scale.
A new cell configuration with a carbon blocking layer placed between a sulfur cathode and separator was demonstrated by Dr Manthiram and colleagues, and showed improved cycling stability. ${ }^{17-20}$ The carbon-based interlayer provided an additional conductive path to the sulfur electrode and efficiently confined the polysulfides on the cathodic side, which enabled a high rate capability and stable cycling performance. However, most studies have only focused on the design of the carbon interlayer to block polysulfides within the cathodic side, and few studies have reported entrapping polysulfides on the anodic side. ${ }^{21,22}$ Even though compact carbon interlayers are located between the sulfur cathode and the separator, moderate amounts of polysulfide can still penetrate the porous interlayer and lead to the deterioration of the $\mathrm{Li}$ anode. ${ }^{3,11,23} \mathrm{In}$ addition, the depletion of the electrolyte is accelerated at high current densities, which could boost the growth of dendritic Li and consequently increase the potential danger of cell failure. Thus, the main challenges to the commercial use of $\mathrm{Li}$ anodes in Li-S batteries are how to effectively prevent the corrosion of the Li metal from polysulfides and control the growth of dendritic and mossy Li.

In this article, we first demonstrate a multifunctional trilayer membrane by sandwiching a conventional polymer separator between the carbon layer and boron nitride $(\mathrm{BN})$ layer to mitigate the diffusion of polysulfides and protect the $\mathrm{Li}$ anode from unexpected side

${ }^{1}$ Department of Energy Engineering, Hanyang University, Seoul, Republic of Korea and ${ }^{2}$ Department of Materials Science and Engineering, University of Maryland, College Park, MD, USA

Correspondence: Professor L Hu, Department of Materials Science and Engineering, University of Maryland, College Park, MD 20742, USA,

E-mail: binghu@umd.edu

or Professor U Paik, Department of Energy Engineering, Hanyang University, Seoul 133-791, Republic of Korea.

E-mail: upaik@hanyang.ac.kr

Received 26 November 2016; revised 31 January 2017; accepted 4 February 2017 
a Sulfur cathode BN-Carbon separator Li metal

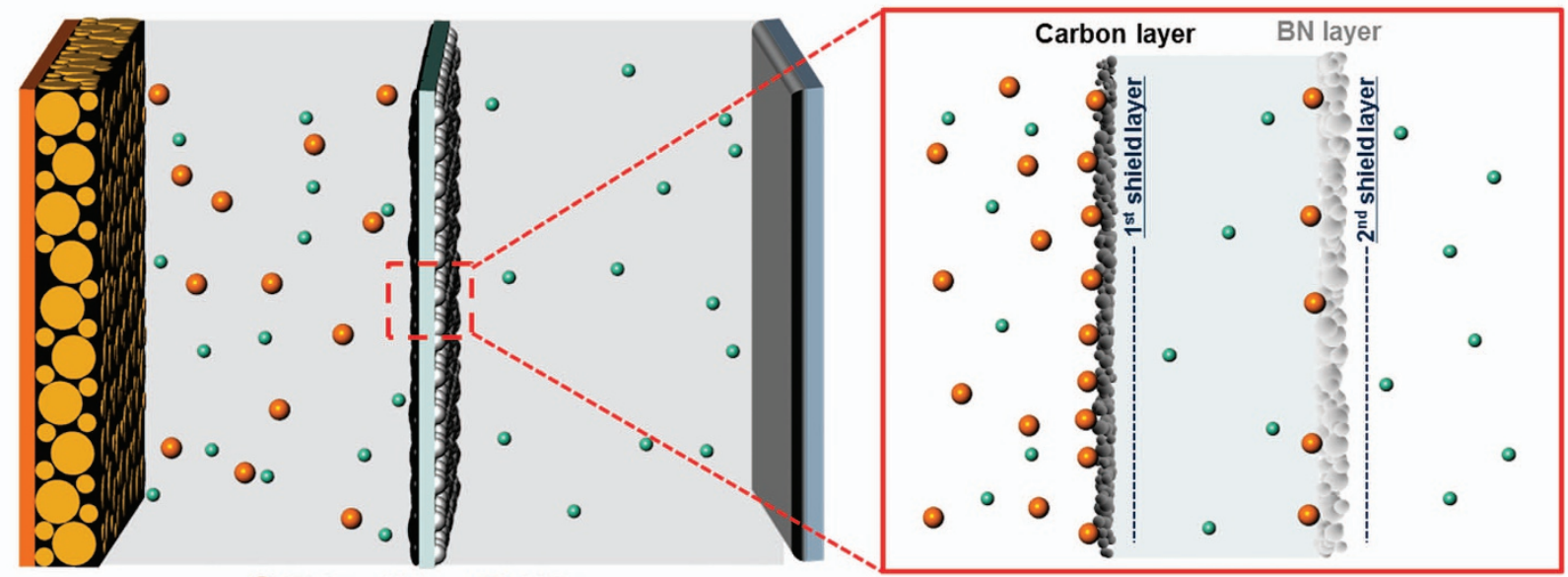

Polysulfide $\odot$ Li ion
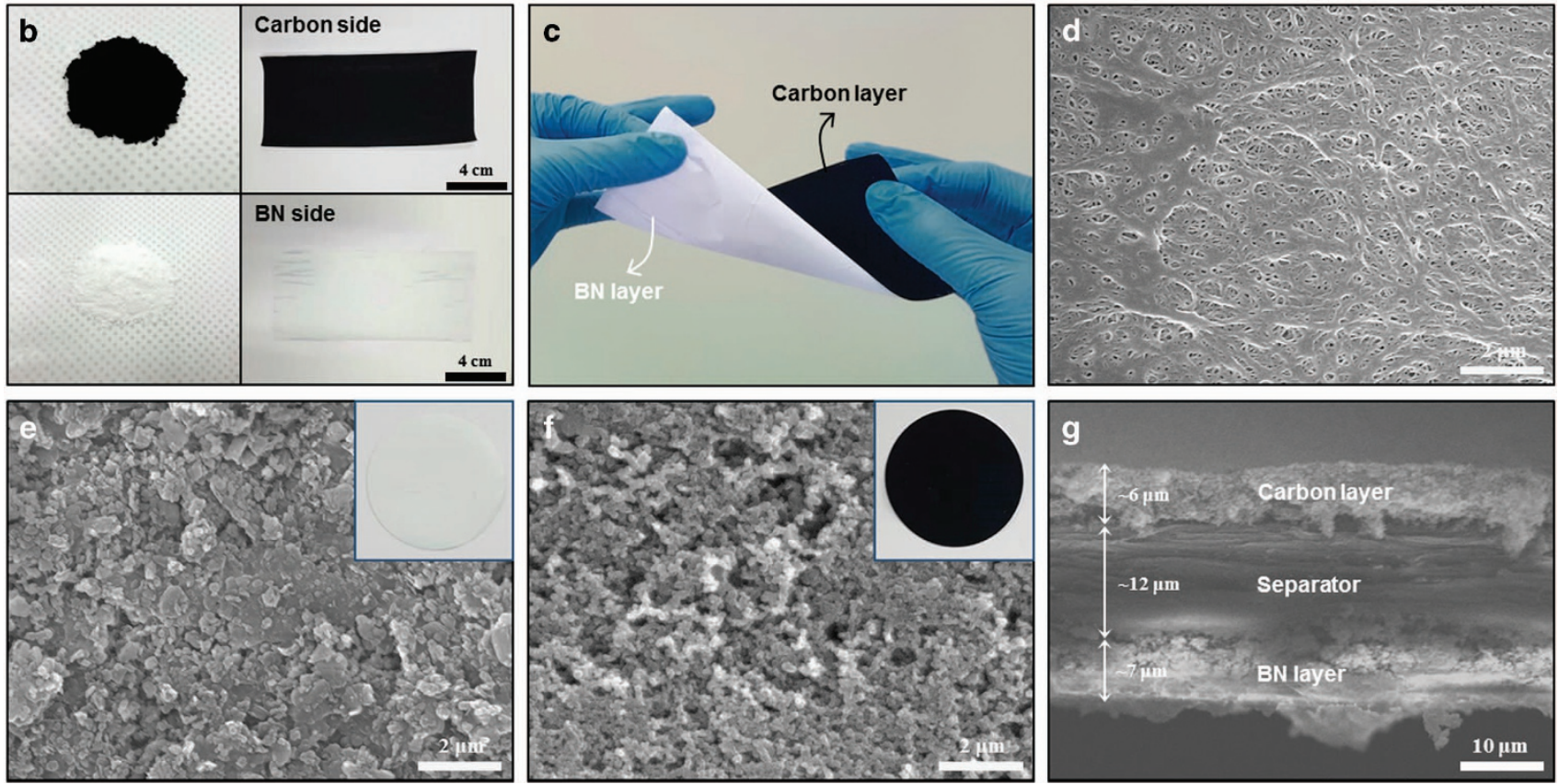

Figure 1 Design of the multifunctional trilayer membrane. (a) Schematic illustration of the systemic role of a boron nitride (BN)-carbon separator in the discharge process; (b) photographs of both nanopowders (carbon, BN) and both sides of a BN-carbon separator; (c) image of a twisted BN-carbon separator; scanning electron microscope (SEM) images of (d) a pristine separator, (e) the BN side and (f) the carbon side of a BN-carbon separator; and (g) crosssectional view of a $\mathrm{BN}$-carbon separator.

reactions. The carbon layer on the sulfur cathode side is used to provide an additional electron pathway and block the dissolved polysulfides. The $\mathrm{BN}$ layer that faces the $\mathrm{Li}$ anode is used to further block the penetrated polysulfides and manipulate the uncontrolled growth of dendritic Li. The multifunctional trilayer membrane, which we called a 'BN-carbon separator', was prepared by casting a carbon ink onto a separator and a $\mathrm{BN}$ ink onto the opposite side of the separator. Carbon nanopowder was used because of its high electrical conductivity and large surface area. ${ }^{24} \mathrm{~A}$ dense layer of carbon nanopowder provides enhanced pathways for rapid electron/ion transport and functions as a first line of defense when polysulfides migrate toward the Li metal. BN nanopowder was used because of its insulating properties and good thermal conductivity. ${ }^{25}$ In a typical cell using a polypropylene separator, solid electrolyte interface layers are easily broken because of the irregular deposition of Li metal and the large-volume expansion of the $\mathrm{Li}$ anode, which leads to the formation of Li dendrites and thus results in short-circuiting and cell failure. ${ }^{25,26}$ As reported in previous research, $\mathrm{BN}$-coated separators have shown significant improvements in the stability of the Li metal anode because of their high thermal conductivity $\left(82 \mathrm{~W} \mathrm{~m}^{-1} \mathrm{~K}\right)$ and minimum Li deposition. ${ }^{25}$ These characteristics enable a conformal thermal distribution of the separator during the discharge-charge process and, thus, contribute to the stable formation of the solid electrolyte interface layer and uniform plating/striping of Li. In addition to its excellent thermal properties, the $\mathrm{BN}$ layer also functions as an additional line of defense to block polysulfides that diffuse through the carbon layer. The polysulfide shuttling effect can be dramatically alleviated by the synergetic combination of the carbon and BN layers, which leads to improved cycling stability of the Li metal anode and enhanced electrochemical performance of the Li-S cell. 

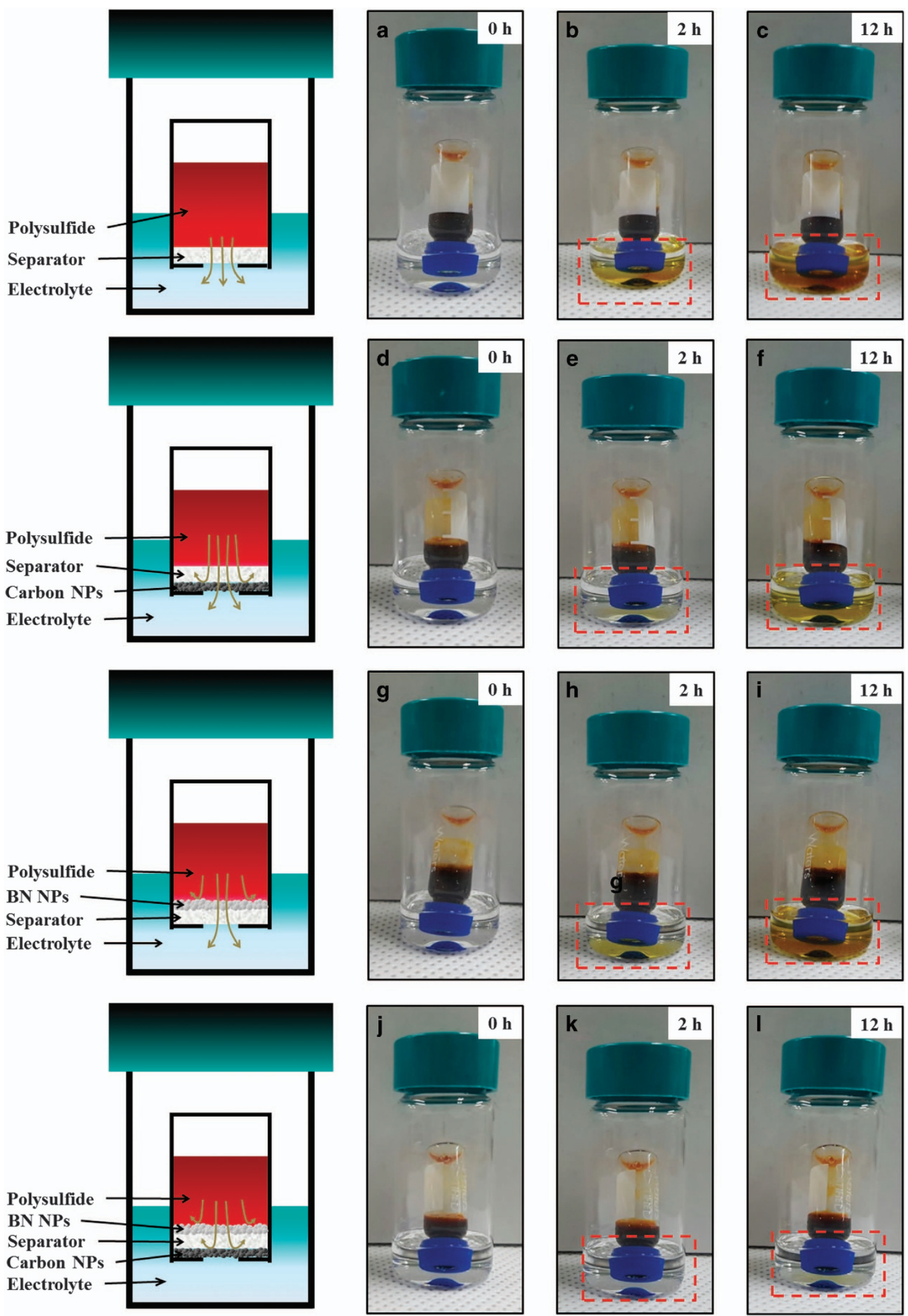

Figure 2 Demonstration of the trilayer membrane to suppress polysulfide. Visual verification of the diffusion test. Each diffusion tester comprised a polysulfide $\left(\mathrm{Li}_{2} \mathrm{~S}_{8}\right)$ solution, a separator and a fresh electrolyte (1 m lithium-bis-trifluoromethylsulfonyl-imide-1,2-dimethoxyethane (DME)-1,3-dioxolane (DIOX)). A pristine separator $(\mathbf{a}-\mathbf{c})$, a carbon separator $(\mathbf{d}-\mathbf{f})$, a BN separator $(\mathbf{g}-\mathbf{i})$ and a BN-carbon separator (j-I) were employed in the diffusion tests. At the beginning, there was no discernible difference between the samples. However, as the elapsed time increased from 0 to $2 \mathrm{~h}$ and then to $12 \mathrm{~h}$, the fresh electrolyte exhibited differences in color because of the different diffusion rates of the polysulfide through the separators. 


\section{EXPERIMENTAL PROCEDURE}

\section{Preparation of the sulfur cathode}

The slurry of the sulfur cathode was prepared by mixing sublimed sulfur $(60 \%)$, super $\mathrm{P}(30 \%)$ and a polyvinylidene fluoride (PVdF) binder $(10 \%)$ in $\mathrm{N}$-methylpyrrolidinone solvent with a planetary mixer (2000 r.p.m., $1 \mathrm{~h}$ ). The slurry was cast in a thick layer onto $\mathrm{Al}$ foil to attain a high sulfur-loading mass. The cast electrode was then dried in a convection oven at $55^{\circ} \mathrm{C}$ overnight to remove the remaining deionized water. After drying, the loading areal density of the sulfur was $2.1 \mathrm{mg} \mathrm{cm}^{-2}$, and the average thickness of the sulfur electrode was $\sim 26 \mu \mathrm{m}$.

\section{Fabrication of the $\mathrm{BN}$-carbon separator}

Carbon ink (or BN ink) was prepared by dispersing a mixture of commercial carbon nanopowder (or BN nanopowder) and polyvinylidene fluoride binder (with a weight ratio of 8:2) into $N$-methylpyrrolidinone solvent. The carbon ink was then cast onto one side of a commercial separator with a doctor blade, and the carbon-casted separator was dried in a convection oven at $50{ }^{\circ} \mathrm{C}$ for $12 \mathrm{~h}$. The BN separator was prepared using the same procedure. The BN-carbon separator was prepared as follows: once the carbon separator was fully dried, the BN ink was then cast onto the opposite side of the carbon layer on the carbon separator with a doctor blade and then dried in a convection oven at $50{ }^{\circ} \mathrm{C}$ for $12 \mathrm{~h}$. Finally, the BN-carbon separator was punched into a disk for use in the cell.

\section{Characterization}

The morphologies of the carbon layer and the $\mathrm{BN}$ layer were analyzed using a field emission scanning electron microscope (JSM-7600F). The galvanostatic discharge-charge evaluations, cycling performances and rate capabilities of the cells were tested with a TOSCAT 3100 battery cycle tester (Toyo Systems, Japan). In addition, a Li metal plating-striping test was performed in $1.0 \mathrm{M}$ $\mathrm{LiPF}_{6}$ ethylene carbonate/diethyl carbonate (EC:DEC $=1: 1$ by volume) and also in different electrolytes, such as $1 \mathrm{~m}$ lithium bis(trifluoromethanesulfonyl)imide in 1,3-dioxolane and 1,2-dimethoxyethane (DIOX:DME $=1: 1$ by volume). The electrochemical impedance spectroscopies of the cells with a pristine separator, a carbon separator, a BN separator and a BN-carbon separator were evaluated using an Autolab PGSTAT 302N potentiostat/galvanostat apparatus (Metrohm AG) at frequencies from 100 to $0.1 \mathrm{~Hz}$ at an excitation amplitude of $5 \mathrm{mV}$. An X-ray photoelectron spectroscopy study was carried out on the Li metal after cycles with a Sigma Probe (Thermo VG Scientific) with Al-K $\alpha \mathrm{X}$-ray radiation. The chemical affinity between the BN powder and polysulfide was tested and is shown in Supplementary Figure S5.

\section{RESULTS AND DISCUSSION}

Figure 1a provides a schematic illustration of the roles of a BN-carbon separator in Li-S batteries. During discharge, polysulfides diffuse out of the sulfur cathode and begin to diffuse toward the Li metal because of the concentration gradient. As the first shield layer, the carbon layer physically blocks the diffusion of the polysulfides. Any polysulfides that diffuse through the carbon layer are further trapped by the $\mathrm{BN}$ layer (the second shield layer). This synergetic design of the trilayer structure facilitates the efficient inhibition of polysulfide shuttling and the reactivation of trapped polysulfides. The $\mathrm{BN}$-carbon separator was prepared by directly casting inks comprising carbon nanopowders and BN nanopowders onto a two-sided polypropylene separator via the slurry coating method, which enabled the large-scale fabrication of a bifacial separator that was $150 \mathrm{~mm}$ long and $60 \mathrm{~mm}$ wide (Figure 1b). Polyvinylidene fluoride binder was added to each casting ink to provide mechanical stability to the casted nanopowders. As shown in Figure 1c, the separator had good mechanical stability and was able to withstand repeated twisting because of the good adhesion between the separator and both nanopowders (carbon and $\mathrm{BN}$ ). Figure $1 \mathrm{~d}$ shows the surface of a pristine polypropylene separator with large pores. After coating $\mathrm{BN}$ and carbon nanopowders onto each side of the separator, the pores were fully covered by $\mathrm{BN}$ nanopowders with a particle size of $\sim 100 \mathrm{~nm}$ (Figure 1e) and by carbon nanopowders with a size of $\sim 50 \mathrm{~nm}$ (Figure 1f). The thicknesses of the BN layer and the carbon layer were $\sim 7$ and $\sim 6 \mu \mathrm{m}$, respectively (Figure $1 \mathrm{~g}$ ).

Figure 2 illustrates the effect of a multifunctional membrane on the suppression of polysulfide diffusion. The diffusion bottles were prepared as in previous studies ${ }^{27,28}$ and placed in fresh electrolyte to compare the diffusion rates of the polysulfides out of the separators. In the bottle containing the pristine separator, the polysulfide solution began to diffuse out of the separator as soon as the diffusion bottle was dipped into the fresh electrolyte (Figure 2a). By contrast, the migration of the polysulfide solution through the different separators (carbon separator, BN separator and BN-carbon separator) was prevented because of physical interception by the nanoparticles. In the bottle with the pristine separator, the fresh electrolyte turned yellow after $2 \mathrm{~h}$ and dark brown after $12 \mathrm{~h}$ because of the rapid diffusion of polysulfides along the concentration gradient (Figure $2 \mathrm{~b}$ and $\mathrm{c}$ ). In contrast, the electrolyte in the bottle with a $\mathrm{BN}$-carbon separator maintained its original color (transparent) for $12 \mathrm{~h}$ (Figure 2j-1); this result directly demonstrated that the $\mathrm{BN}$-carbon separator was effective at inhibiting the diffusion of polysulfides. In each bottle with a carbon separator (Figure $2 \mathrm{~d}$ ) and a $\mathrm{BN}$ separator (Figure $2 \mathrm{~g}$ ), the fresh electrolyte began to turn light yellow after only $2 \mathrm{~h}$ (Figure $2 \mathrm{e}$ and $\mathrm{h}$ ) and dark yellow after $12 \mathrm{~h}$ (Figure $2 \mathrm{f}$ and $\mathrm{i}$ ), which implied that both separators curbed polysulfide shutting, but to a lesser extent compared with the $\mathrm{BN}$-carbon separator. Based on the colors of the fresh electrolytes, the diffusion rates of the polysulfides through each separator were ascertained as follows: pristine separator $>\mathrm{BN}$ separator $>$ carbon separator $>\mathrm{BN}$-carbon separator. It can be inferred that the diffusion rates of the polysulfides through each separator were inversely proportional to the cycling performance of each electrode, which is consistent with the obtained results.

The electrochemical performances of the Li-S cells with the pristine separator, the carbon separator, the $\mathrm{BN}$ separator and the BN-carbon separator are shown in Figure 3. All of the cells were assembled in an argon-filled glovebox and packaged in coin cells. To demonstrate the blocking effect of the trilayer membrane, a fresh $1 \mathrm{M}$ lithium-bistrifluoromethylsulfonyl-imide-DME-DIOX electrolyte without $\mathrm{LiNO}_{3}$ additive was used to evaluate the electrochemical performance of the Li-S cells. The initial discharge-charge curves of each electrode at $0.5 \mathrm{C}$ are shown in Figure 3a. The sulfur cathode with the pristine separator delivered a discharge capacity of $772.0 \mathrm{mAhg}^{-1}$ and a charge capacity of $961.5 \mathrm{mAhg}^{-1}$, which represents a severe polysulfide-shutting effect. When the carbon separator and the BN-carbon separator were placed in the Li-S cell, the discharge capacities of the cell increased to $921.1 \mathrm{mAh} \mathrm{g}^{-1}$ and $1018.5 \mathrm{mAh} \mathrm{g}^{-1}$ (sulfur utilization: 60.8\%), respectively, which was attributed to the improved utilization of active materials through an additional current collector. However, the main difference between the electrodes was that the sulfur cathode with the carbon separator had a higher charge capacity $\left(1049.8 \mathrm{mAh} \mathrm{g}^{-1}\right)$ than the sulfur cathode with the $\mathrm{BN}$-carbon separator $\left(1036.4 \mathrm{mAh}^{-1}\right)$, indicating poorer blocking of the polysulfide shuttling effect by the carbon separator than that of the $\mathrm{BN}$-carbon separator. No significant improvement was observed in the electrochemical performance of the sulfur cathode with the $\mathrm{BN}$ separator, even compared with the sulfur cathode with the pristine separator.

The cycling performances of the electrodes were evaluated at $0.5 \mathrm{C}$ and are shown in Figure 3b. After 250 cycles, the sulfur cathode with the pristine separator retained a discharge capacity of 
a
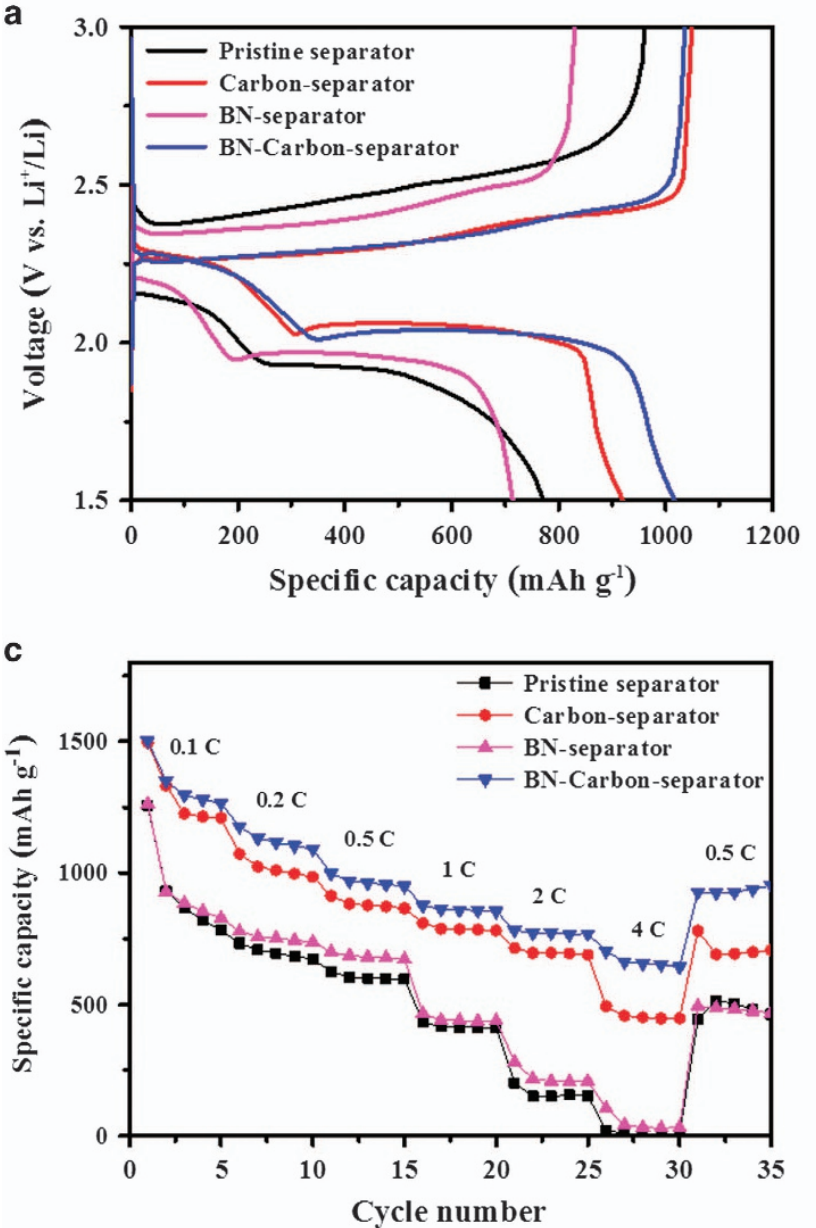

b

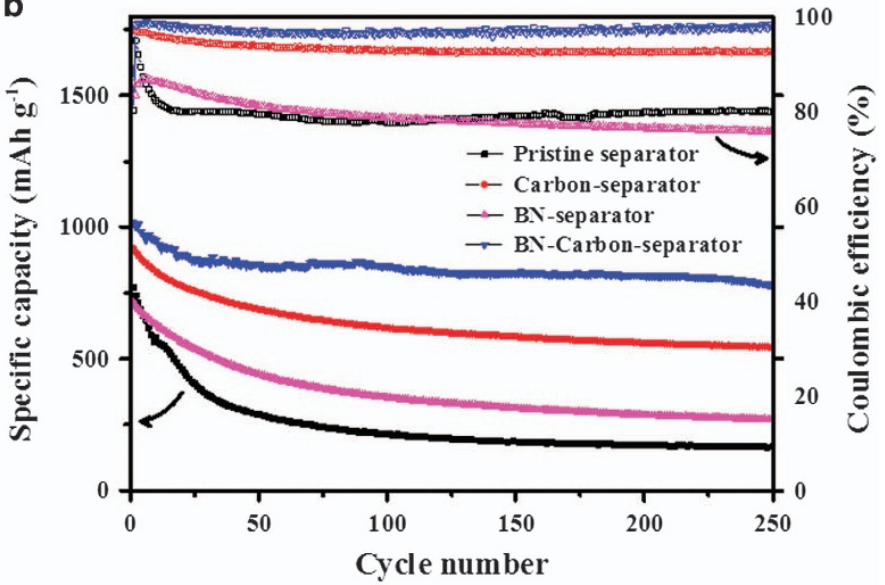

d

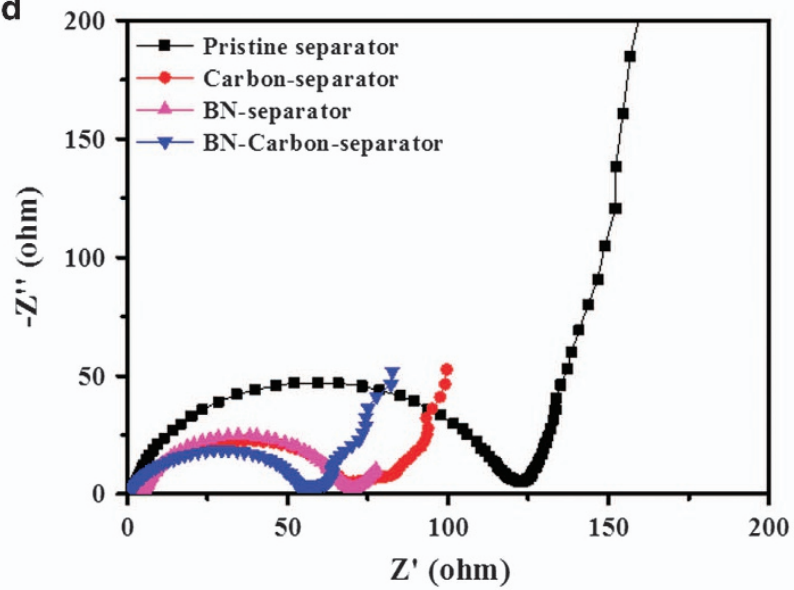

Figure 3 Electrochemical characterization. Electrochemical performances of sulfur cathode cells with a pristine separator, carbon separator, boron nitride (BN) separator and BN-carbon separator. (a) Initial discharge/charge curves (at $0.5 \mathrm{C}$ ), (b) cycle performances (at $0.5 \mathrm{C}$ ), (c) rate capabilities and (d) Nyquist plots of each electrode.

166.2 $\mathrm{mAh} \mathrm{g}^{-1}$, which was a retention of $21.5 \%$. The irreversible and rapid capacity fade was due to the severe loss of sulfur from the cathode electrode. When the carbon separator and the BN-carbon separator were placed in the Li-S cell, the capacity retentions of both cells improved because dissolved polysulfides were confined within the cathodic side and reactivated as active materials during cycling. ${ }^{24,29}$ To provide practical information about the sulfur electrodes, the gravimetric and volumetric energy densities of each electrode were calculated and are shown in Supplementary Figure S1.

The sulfur cathode with the BN-carbon separator exhibited a higher discharge capacity of $780.7 \mathrm{mAh} \mathrm{g}^{-1}$ with a higher retention $(76.6 \%)$ than the sulfur cathode with the carbon separator $\left(545.3 \mathrm{mAh} \mathrm{g}^{-1}, 59.2 \%\right)$ because of the significant suppression of polysulfide leakage by the multifunctional separator and the increased electrochemical utilization of sulfur. To investigate the systemic benefit of a $\mathrm{BN}$-carbon separator on the rate capabilities, each electrode was evaluated at current densities from 0.1 to $4 \mathrm{C}$ (Figure 3c). Given the inherently poor electric conductivity of sulfur, the sulfur cathode with the pristine separator showed poor rate capabilities at high current densities $(2-4 \mathrm{C})$. When the conductive carbon separator and the $\mathrm{BN}$-carbon separator were employed, the additional electron path led to increased reutilization of the trapped polysulfide species and facilitated high electrochemical reversibility, which resulted in high discharge capacities even at high current densities (2-4 C). The sulfur cathode with the BN-carbon separator delivered an extremely high specific capacity of $702.5 \mathrm{mAhg}^{-1}$ at $4 \mathrm{C}$, whereas the sulfur cathodes with the pristine separator and the BN separator exhibited poor specific capacities of $22.5 \mathrm{mAh} \mathrm{g}^{-1}$ and 105.4 $\mathrm{mAhg}^{-1}$, respectively. The outstanding cycling performances and rate capabilities of the sulfur cathode with the BN-carbon separator were mainly attributed to the systemic advantages of the carbon layer and the BN layer: (a) the carbon layer suppresses polysulfide leakage from the cathodic side and reactivates the trapped active materials, and (b) the $\mathrm{BN}$ layer further reduces the migration of dissolved polysulfides and facilitates a stable electrochemical reaction. These two synergistic contributions effectively curb the diffusion of polysulfides toward the Li metal, which protects it from side reactions.

To ascertain the interfacial behaviors of the electrodes when modified separators were introduced, electrochemical impedance spectroscopy experiments were performed at frequencies of $100 \mathrm{kHz}$ to $0.1 \mathrm{~Hz}$ with an amplitude of $5 \mathrm{mV}$ (Figure 3d). The intersection of the $\mathrm{X}$-axis and the semicircle in Figure $3 \mathrm{~d}$ indicates an Ohmic resistance between the electrode and the electrolyte. ${ }^{30}$ The Ohmic resistances of the sulfur cathodes with the pristine separator, the carbon separator, the $\mathrm{BN}$ separator and the BN-carbon separator were $1.92,2.68,5.12$ and $1.67 \Omega$, respectively. 

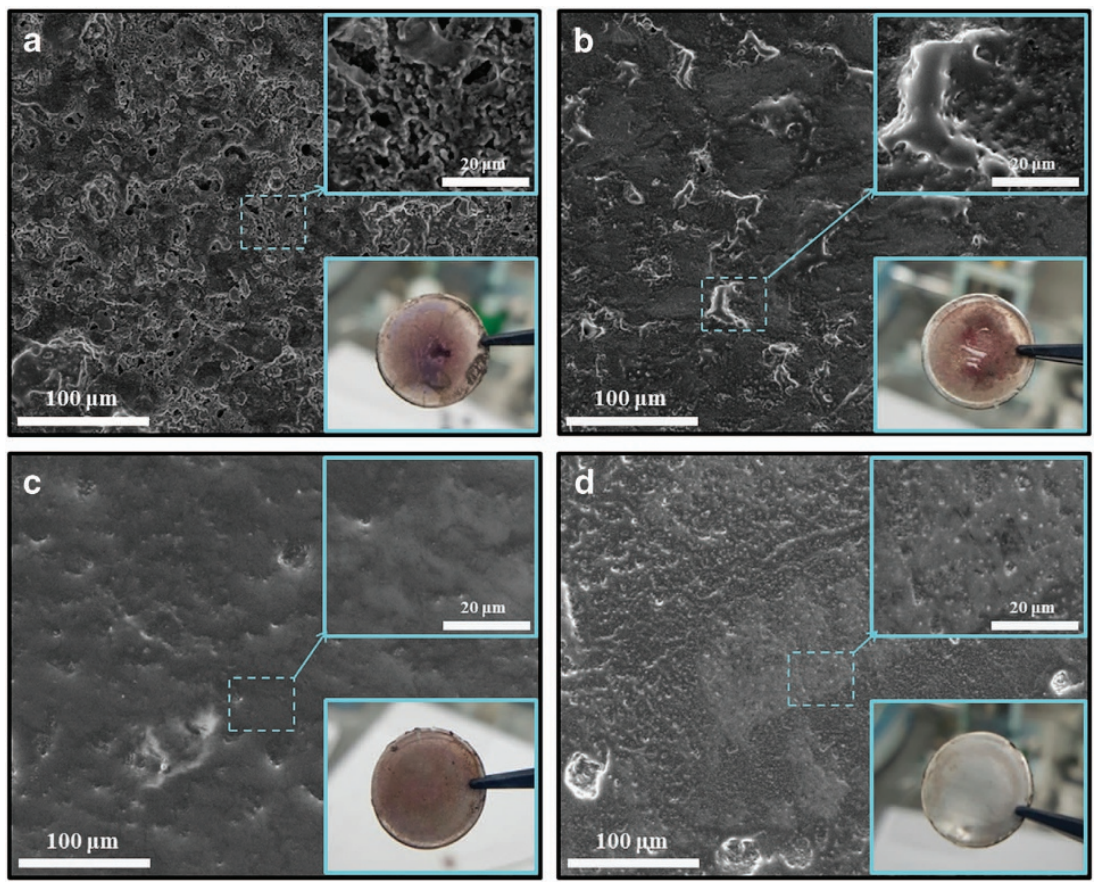

Figure 4 Morphological characterizations of cycled lithium metal. Photographs and morphologies after 250 cycles: the lithium metals of sulfur cells with (a) a pristine separator, (b) a carbon separator, (c) a BN separator and (d) a BN-carbon separator. The lithium metal was approximately $230 \mu \mathrm{m}$ thick.

The diameters of the semicircles in Figure 3d, which are associated with charge transfer resistance, differed when the modified separators were introduced. The sulfur cathode with the BN-carbon separator had the lowest polarization resistance $(55.1 \Omega$ ) compared with the sulfur cathodes with the pristine separator $(122.9 \Omega)$, the carbon separator $(69.4 \Omega)$ and the $\mathrm{BN}$ separator $(70.5 \Omega)$. The improved surface reaction kinetics were mainly attributed to the diminished polysulfide shuttling effect and the increased reutilization of dissolved sulfur and polysulfide intermediates. ${ }^{24,29}$

A cyclic voltammetry study was conducted at a scan rate of $0.2 \mathrm{mV} \mathrm{s}^{-1}$ to explore the electrochemical stabilities during repetitive cycles (Supplementary Figure S2). Each electrode displayed typical cyclic voltammetry shapes of a Li-S cell: two cathodic peaks at approximately 2.28 and $1.97 \mathrm{~V}$, which were associated with the electrochemical reaction of $\mathrm{S}_{8}$ to long chains $\left(\mathrm{Li}_{2} \mathrm{~S}_{\mathrm{n}} 4 \leqslant n \leqslant 8\right)$ and then to $\mathrm{Li}_{2} \mathrm{~S}_{2} / \mathrm{Li}_{2} \mathrm{~S}$, respectively, and one anodic peak at $2.46 \mathrm{~V}$, which was related to the conversion of polysulfide species to $\mathrm{S}_{8}{ }^{29}$ The cyclic voltammetry curve of the sulfur cathode with the BN-carbon separator exhibited sharp discharge/charge peaks at approximately redox potentials and no differences in the potential position and intensity change even during repetitive cycles, whereas the other electrodes showed poor reversibility or low current intensity. These results directly verified that the BN-carbon separator facilitated the efficient reaction and electrochemical reversibility of the sulfur cathode. ${ }^{22}$

To understand the capacity fade mechanism of the electrodes, the cells were disassembled after 200 cycles and investigated (Figure 4). In the sulfur cathode with the pristine separator, the Li metal was covered by many black spots. An scanning electron microscope (SEM) study was performed to observe the microstructure of these spots. As shown in the inset of Figure 4a, the spots comprised interconnected particles with diameters of $5-10 \mu \mathrm{m}$ that were distributed over the entire area of the Li metal. To characterize the black spots, an $\mathrm{X}$-ray photoelectron spectroscopy analysis was carried out (Suppleme- ntary Figure S3). The X-ray photoelectron spectroscopy spectrum of the black spots for $\mathrm{S} 2 \mathrm{p}$ was divided into two peaks, centered at $\mathrm{S} 2 \mathrm{p}_{3 / 2}(162.2 \mathrm{eV})$ and $\mathrm{S} 2 \mathrm{p}_{3 / 2}(160.9 \mathrm{eV})$, which corresponded to $\mathrm{Li}_{2} \mathrm{~S}_{2}$ and $\mathrm{Li}_{2} \mathrm{~S}$, respectively. ${ }^{31,32}$ By contrast, the Li metal collected from the cell of the sulfur cathode with the BN-carbon separator showed a clean and shiny metal surface (inset of Figure 4d). This implied that the insoluble polysulfides $\left(\mathrm{Li}_{2} \mathrm{~S}_{2}\right.$ and $\mathrm{Li}_{2} \mathrm{~S}$ ) accumulated less on the surface of the $\mathrm{Li}$ metal because of the effective suppression of polysulfides by the BN-carbon separator, which was also confirmed by the SEM image (Figure 4d). The Li metal that was collected from the cells with the carbon separator and BN separator had more intact surfaces than the Li metal collected from the cell (the sulfur cathode with the pristine separator); however, the deposited polysulfides could be distinguished from the $\mathrm{Li}$ metal surface (Figure $4 \mathrm{~b}$ and $\mathrm{c}$ ), which indicated a relatively low effectiveness at protecting the Li metal from polysulfide species.

As discussed above, the BN-carbon separator demonstrated excellent performance in diminishing the migration of polysulfides toward the Li metal, which protected the $\mathrm{Li}$ anode from soluble polysulfide species. Li dendrites were not observed on the surface of the Li metal, which was in agreement with the report that polysulfides can influence the formation of dendritic lithium. ${ }^{33}$ To validate the substantial protective effect of the $\mathrm{BN}$-carbon separator on the growth of $\mathrm{Li}$ dendrites with a different approach, we further performed a $\mathrm{Li}$ metal plating-striping test in an $\mathrm{Li} / \mathrm{Cu}$ cell, in which $\mathrm{Cu}$ foil was used as a working electrode, $1.0 \mathrm{M} \mathrm{LiPF} 6$ in ethylene carbonate/diethyl carbonate (EC:DEC $=1: 1$ by volume) was used as an electrolyte, and Li metal was used as a reference electrode. ${ }^{4,10}$ Figure 5 shows the potential profile and cycling performance of each electrode at a high areal current density of $1 \mathrm{~mA} \mathrm{~cm}^{-1}$. The cells with the $\mathrm{BN}$ separator and the $\mathrm{BN}$-carbon separator showed significant improvements in cycling performance (Figure 5a). The Coulombic efficiencies of both cells were maintained at $\sim 85 \%$ 

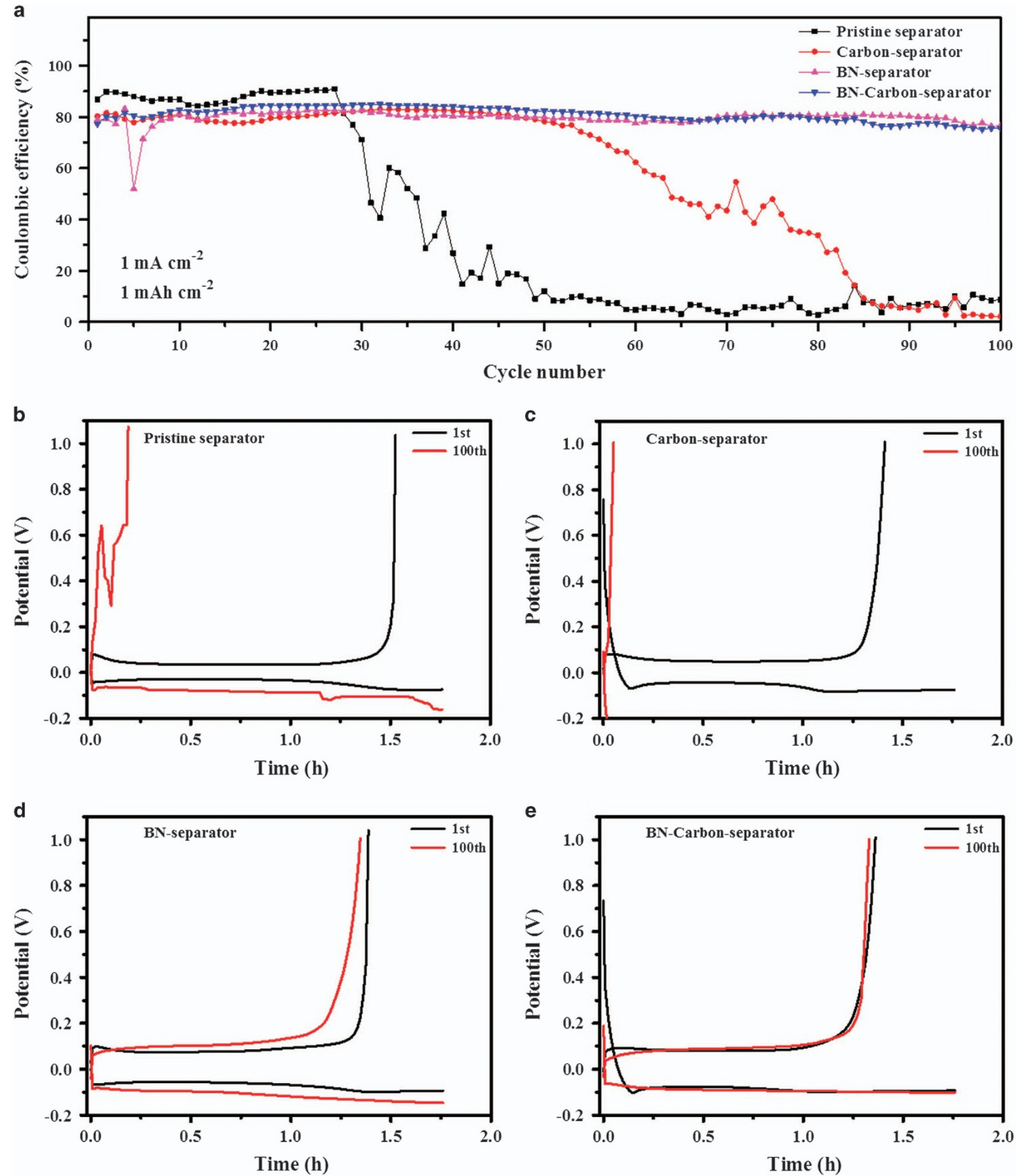

Figure 5 Electrochemical performances of $\mathrm{Li}-\mathrm{Cu}$ cells with a pristine separator, a carbon separator, a BN separator and a BN-carbon separator. (a) Comparison of cycle performances of each electrode and the voltage profiles of the sulfur cell with (b) a pristine separator, (c) a carbon separator, (d) a BN separator and (e) a BN-carbon separator for the first and 100th cycle. All of the electrodes were tested at a current density of $1 \mathrm{~mA} \mathrm{~cm}^{-1}$. The Li deposition capacity was set to $1 \mathrm{mAh} \mathrm{cm}^{-1}$, and the cutoff voltage for the striping was set to $1.0 \mathrm{~V}$.

for 100 cycles with no decay. In contrast, the cell with the pristine separator showed an abrupt fade in Coulombic efficiency after 27 cycles, and the cell with the carbon separator exhibited a gradual fade in Coulombic efficiency after 50 cycles. As shown in Figure 5b-e, the cells with each separator showed rapid potential decreases below $0.0 \mathrm{~V} \mathrm{vs} \mathrm{Li}^{+} / \mathrm{Li}$ as soon as the current was applied, which indicated $\mathrm{Li}$ deposition on the $\mathrm{Cu}$ foil. In the initial cycle, the potential profiles of each electrode showed similar electrochemical behavior to 
previous reports. ${ }^{25,26}$ At 100 cycles, the cells with the pristine separator and the carbon separator showed a fatal decay of the Li metal striping test, while the cells with the $\mathrm{BN}$ separator and the $\mathrm{BN}$-carbon separator exhibited stable Li striping processes. Comparing the cell potential profile of the $\mathrm{BN}$ separator with that of the $\mathrm{BN}$-carbon separator, the voltage curves of the $\mathrm{BN}$-carbon separator at the first and 100th cycles showed more consistent behaviors in terms of the Coulombic efficiency and reaction potentials than those of the $\mathrm{BN}$ separator; this demonstrated the superiority of the $\mathrm{BN}$-carbon separator in Li metal protection. To provide evidence that the $\mathrm{BN}$-carbon separator facilitated stable cycling performance and Coulombic efficiency of the Li metal, ex situ SEM observations were carried out on the $\mathrm{Cu}$ foil after 100 cycles (Supplementary Figure S4). In the cells with the pristine separator (Supplementary Figure S4a) and the carbon separator (Supplementary Figure S4b), many one-dimensional $\mathrm{Li}$ wires with diameters of $200 \mathrm{~nm}$ had grown directly on the $\mathrm{Cu}$ foil. When the $\mathrm{BN}$ separator was employed, $\mathrm{Li}$ granules with a larger diameter of $1 \mu \mathrm{m}$ formed on the $\mathrm{Cu}$ foil because fewer nuclei formed during $\mathrm{Li}$ deposition, which was consistent with the results of previous studies (Supplementary Figure S4c). ${ }^{25,26}$ Especially with the $\mathrm{BN}$-carbon separator, the Li was uniformly deposited with no cracks or sharp pillars on the $\mathrm{Cu}$ foil, which could decrease the risk of short-circuiting within the cell and improve the cycling stability of the Li metal anode (Supplementary Figure S4d).

\section{CONCLUSIONS}

In this study, we designed a multifunctional trilayer membrane (BN-carbon separator) to efficiently suppress the polysulfide shuttling effect and improve the electrochemical performance of $\mathrm{Li}-\mathrm{S}$ cells. When a BN-carbon separator was employed, the sulfur cathode delivered a high initial discharge capacity of $1018.5 \mathrm{mAh} \mathrm{g}^{-1}$ at $0.5 \mathrm{C}$ and retained its capacity of $780.7 \mathrm{mAh} \mathrm{g}^{-1}$ over 250 cycles. In addition, the Li metal in the anode was effectively protected from polysulfide species during the cycles, which led to the stable operation of the $\mathrm{Li}$ anode with excellent cycle retention. The improved electrochemical performance of the Li-S cell with the BN-carbon separator is attributed to three main advantages: (a) the efficient suppression of polysulfides within the cathodic side, (b) an additional electron pathway (imparted by the carbon layer) and (c) a uniform thermal distribution of the separator (imparted by the $\mathrm{BN}$ layer). Unlike previous approaches to address the systemic issues of Li-S cells, the concept of employing a multifunctional $\mathrm{BN}$-carbon separator represents a novel strategy to improve the electrochemical performance of $\mathrm{Li}-\mathrm{S}$ cells and protect the Li metal from side reactions. Moreover, the multifunctional membrane showed promising results in achieving stable operation of the Li metal even at high current densities; this opens a new avenue to address the practical issues underlying the use of Li metal as an anode electrode.

\section{CONFLICT OF INTEREST}

The authors declare no conflict of interest.

\section{ACKNOWLEDGEMENTS}

This work was supported by the Korea Institute of Energy Technology Evaluation and Planning (KETEP), and the Ministry of Trade, Industry \& Energy (MOTIE) of the Republic of Korea (No. 20168510050080) and the Energy Efficiency \& Resources Core Technology Program of the Korea Institute of Energy Technology Evaluation and Planning (KETEP), which granted financial resources from the Ministry of Trade, Industry \& Energy, Republic of Korea (No. 20142020104190).

1 Ji, X., Lee, K. T. \& Nazar, L. F. A highly ordered nanostructured carbon-sulphur cathode for lithium-sulphur batteries. Nat. Mater. 8, 500-506 (2009).

$2 \mathrm{Ji}$, X. \& Nazar, L. F. Advances in Li-S batteries. J. Mater. Chem. 20, 9821-9826 (2010).

3 Bresser, D., Passerini, S. \& Scrosati, B. Recent progress and remaining challenges in sulfur-based lithium secondary batteries - a review. Chem. Commun. 49, 10545-10562 (2013).

4 Manthiram, A., Fu, Y. Z., Chung, S. H., Zu, C. X. \& Su, Y. S. Rechargeable lithium-sulfur batteries. Chem. Rev. 114, 11751-11787 (2014).

5 Yin, Y. X., Xin, S., Guo, Y. G. \& Wan, L. J. Lithium-sulfur batteries: electrochemistry, materials, and prospects. Angew. Chem. Int. Ed. 52, 13186-13200 (2013).

6 Chen, R. J., Zhao, T. \& Wu, F. From a historic review to horizons beyond: lithium-sulphur batteries run on the wheels. Chem. Commun. 51, 18-33 (2015).

7 Park, J., Moon, J., Kim, C., Kang, J. H., Lim, E., Park, J., Lee, K. J., Yu, S. H., Seo, J. H., Lee, J., Heo, J., Tanaka, N., Cho, S. P., Pyun, J., Cabana, J., Hong, B. H. \& Sung, Y. E. Graphene quantum dots: structural integrity and oxygen functional groups for high sulfur/sulfide utilization in lithium sulfur batteries. NPG Asia Mater. 8, e272 (2016)

8 Li, G., Wang, C., Cai, W., Lin, Z., Li, Z. \& Zhang, S. The dual actions of modified polybenzimidazole in taming the polysulfide shuttle for long-life lithium-sulfur batteries. NPG Asia Mater. 8, e317 (2016).

9 Fu, Y. Z., Su, Y. S. \& Manthiram, A. Sulfur-carbon nanocomposite cathodes improved by an amphiphilic block copolymer for high-rate lithium-sulfur batteries. ACS Appl. Mater. Interfaces 4, 6046-6052 (2012).

10 Xu, T., Song, J. X., Gordin, M. L., Sohn, H., Yu, Z. X., Chen, S. R. \& Wang, D. H. Mesoporous carbon-carbon nanotube-sulfur composite microspheres for high-arealcapacity lithium-sulfur battery cathodes. ACS Appl. Mater. Interfaces 5, 11355-11362 (2013).

11 Zhang, Q., Cheng, X. B., Huang, J. Q., Peng, H. J. \& Wei, F. Review of carbon materials for advanced lithium-sulfur batteries. New Carbon Mater. 29, 241-264 (2014).

12 Kim, J. H., Fu, K., Choi, J., Kil, K., Kim, J., Han, X., Hu, L. \& Paik, U. Encapsulation of S/SWNT with PANI web for enhanced rate and cycle performance in lithium sulfur batteries. Sci. Rep. 5, 8946 (2015).

13 Li, L., Ruan, G., Peng, Z., Yang, Y., Fei, H., Raji, A. R. O., Samuel, E. L. \& Tour, J. M. Enhanced cycling stability of lithium sulfur batteries using sulfur polyaniline-graphene nanoribbon composite cathodes. ACS Appl. Mater. Interfaces 6, 15033-15039 (2014).

14 Tang, Q., Shan, Z., Wang, L., Qin, X., Zhu, K., Tian, J. \& Liu, X. Nafion coated sulfur-carbon electrode for high performance lithium-sulfur batteries. J. Power Sources 246, 253-259 (2014).

15 Yang, Y., Yu, G., Cha, J. J., Wu, H., Vosgueritchian, M., Yao, Y., Bao, Z. \& Cui, Y. Improving the performance of lithium-sulfur batteries by conductive polymer coating. ACS Nano 5, 9187-9193 (2011).

16 Kim, J. H., Fu, K., Choi, J., Sun, S., Kim, J., Hu, L. \& Paik, U. Hydroxylated carbon nanotube enhanced sulfur cathodes for improved electrochemical performance of lithium-sulfur batteries. Chem. Commun. 51, 13682-13685 (2015)

17 Chung, S. H. \& Manthiram, A. Carbonized eggshell membrane as a natural polysulfide reservoir for highly reversible Li-S batteries. Adv. Mater. 26, 1360-1365 (2014).

18 Singhal, R., Chung, S. H., Manthiram, A. \& Kalra, V. A free-standing carbon nanofiber interlayer for high-performance lithium-sulfur batteries. J. Mater. Chem. A 3, 4530-4538 (2015).

$19 \mathrm{Su}$, Y. S. \& Manthiram, A. A new approach to improve cycle performance of rechargeable lithium-sulfur batteries by inserting a free-standing MWCNT interlayer. Chem. Commun. 48, 8817-8819 (2012).

20 Zu, C. X., Su, Y. S., Fu, Y. Z. \& Manthiram, A. Improved lithium-sulfur cells with a treated carbon paper interlayer. Phys. Chem. Chem. Phys. 15, 2291-2297 (2013)

21 Huang, C., Xiao, J., Shao, Y., Zheng, J., Bennett, W. D., Lu, D., Saraf, L. V., Engelhard, M., Ji, L., Zhang, J. \& Li, X. Manipulating surface reactions in lithium-sulphur batteries using hybrid anode structures. Nat. Commun. 5, 3015 (2014).

22 Wu, F., Ye, Y., Chen, R., Qian, J., Zhao, T., Li, L. \& Li, W. Systematic effect for an ultra long cycle lithium-sulfur battery. Nano Lett. 15, 7431-7439 (2015).

23 Bauer, I., Thieme, S., Bruckner, J., Althues, H. \& Kaskel, S. Reduced polysulfide shuttle in lithium-sulfur batteries using Nafion-based separators. J. Power Sources 251, 417-422 (2014).

24 Yao, H., Yan, K., Li, W., Zheng, G., Kong, D., Seh, Z. W., Narasimhan, V. K., Liang, Z. \& Cui, Y. Improved lithium-sulfur batteries with a conductive coating on the separator to prevent the accumulation of inactive S-related species at the cathode-separator interface. Energy Environ. Sci. 7, 3381-3390 (2014).

25 Luo, W., Zhou, L., Fu, K., Yang, Z., Wan, J., Manno, M., Yao, Y., Zhu, H., Yang, B. \& Hu, L. A thermally conductive separator for stable Li metal anodes. Nano Lett. 15, 6149-6154 (2015).

26 Zheng, G., Lee, S. W., Liang, Z., Lee, H. W., Yan, K., Yao, H., Wang, H., Li, W., Chu, S. $\&$ Cui, Y. Interconnected hollow carbon nanospheres for stable lithium metal anodes. Nat. Nanotechnol. 9, 618-623 (2014). 
27 Yang, Y., Zheng, G. Y. \& Cui, Y. A membrane-free lithium/polysulfide semi-liquid battery for large-scale energy storage. Energy Environ. Sci. 6, 1552-1558 (2013).

28 Kim, J. H., Seo, J., Choi, J., Shin, D., Carter, M., Jeon, Y., Wang, C., Hu, L. \& Paik, U. Synergistic ultrathin functional polymer-coated carbon nanotube interlayer for high performance lithium-sulfur batteries. ACS Appl. Mater. Interfaces 8, 20092-20099 (2016).

29 Chung, S. H. \& Manthiram, A. A polyethylene glycol-supported microporous carbon coating as a polysulfide trap for utilizing pure sulfur cathodes in lithium-sulfur batteries. Adv. Mater. 26, 7352-7357 (2014).

30 Kim, J. H., Lee, S., Lee, J. W., Song, T. \& Paik, U. 3D-interconnected nanoporous RGO-CNT structure for supercapacitors application. Electrochim. Acta 125, 536-542 (2014).

31 Demir-Cakan, R., Morcrette, M., Guéguen, A., Dedryvère, R. \& Tarascon, J. M. Li-S batteries: simple approaches for superior performance. Energy Environ. Sci. 6, 176-182 (2013).

32 Helen, M., Reddy, M. A., Diemant, T., Golla-Schindler, U., Behm, R. J., Kaiser, U. \& Fichtner, M. Single step transformation of sulphur to Li2S2/Li2S in Li-S batteries. Sci. Rep. 5, 12146 (2015).
33 Li, W., Yao, H., Yan, K., Zheng, G., Liang, Z., Chiang, Y. M. \& Cui, Y. The synergetic effect of lithium polysulfide and lithium nitrate to prevent lithium dendrite growth Nat. Commun. 6, 7436 (2015).

(c) (i) This work is licensed under a Creative Commons Attribution 4.0 International License. The images or other third party material in this article are included in the article's Creative Commons license, unless indicated otherwise in the credit line; if the material is not included under the Creative Commons license, users will need to obtain permission from the license holder to reproduce the material. To view a copy of this license, visit http:// creativecommons.org/licenses/by/4.0/

(C) The Author(s) 2017

Supplementary Information accompanies the paper on the NPG Asia Materials website (http://www.nature.com/am) 